\title{
Results of a proof of concept, double-blind, randomized trial of a second generation antisense oligonucleotide targeting high-sensitivity C-reactive protein (hs-CRP) in rheumatoid arthritis
}

\author{
Marshelle S Warren', Steven G Hughes ${ }^{1 *}$, Walter Singleton ${ }^{1}$, Mason Yamashita ${ }^{1}$ and Mark C Genovese ${ }^{2}$
}

\begin{abstract}
Introduction: This randomized, double-blind, phase II study evaluated the pharmacodynamics, safety and tolerability of ISIS 329993 (ISIS-CRP $P_{\mathrm{Rx}}$ ), an antisense oligonucleotide, in patients with active rheumatoid arthritis (RA).

Methods: Patients with active RA of at least six months duration were randomized into three cohorts to receive ISIS-CRP Rx (100 mg, 200 mg or 400 mg) or placebo (3 active:1 placebo within each cohort) via subcutaneous (SC) injection on Days 1, 3, 5 and 8 and then once weekly for the next 11 weeks. The effects of study treatment on high-sensitivity C-reactive protein (hs-CRP) level were evaluated. An exploratory analysis on disease activity was assessed via the American College of Rheumatology 20\% improvement criteria (ACR20). Safety was evaluated via adverse events and laboratory measures.
\end{abstract}

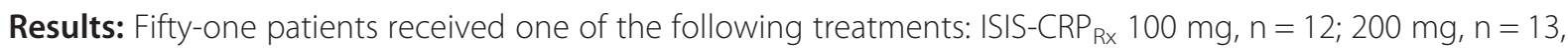
$400 \mathrm{mg}, \mathrm{n}=14$; placebo $\mathrm{n}=12$. In the ISIS-CRP $\mathrm{Rx}_{\mathrm{x}}$ treatment groups there were dose-dependent reductions in hs-CRP. At Day 36 the mean percent change from baseline was: placebo: -14.4\%; ISIS-CRP 100 mg: -19.5\%; $200 \mathrm{mg}:-56.6 \%$ and $400 \mathrm{mg}:-76.7 \%$, ( $P=0.0015$ placebo compared to $400 \mathrm{mg}$ ). There were no differences between treatment groups and placebo in the ACR20 at Day 36 or Day 92. There were no serious infections and no elevations in liver function tests, lipids, creatinine or other lab abnormalities related to ISIS-CRP Rx.

Conclusions: In this study, ISIS-CRP Rx selectively reduced hs-CRP in a dose-dependent manner, and was well-tolerated in patients with RA. Its utility as a therapy in RA remains unclear.

Trial registration: Clinicaltrials.gov NCT01414101. Registered 21 July 2011.

\section{Introduction}

Studies of patients with rheumatoid arthritis (RA) document a correlation between C-reactive protein (CRP) blood concentration and worsening of RA symptoms. Inflammation in RA is closely related to the production of CRP and proinflammatory cytokines [1]. Levels of CRP correlate closely with changes in inflammation/disease activity, radiological damage and progression, and functional disability [1]. Although CRP is considered to be a marker of inflammation in RA, it may also function in the promotion of inflammation through complement activation [2].

\footnotetext{
* Correspondence: shughes@isisph.com

'Isis Pharmaceuticals, Inc, 2855 Gazelle Court, Carlsbad, CA 92010, USA Full list of author information is available at the end of the article
}

CRP, and in particular the high-sensitivity CRP (hs-CRP) assay, has been shown to be more closely associated with disease activity variables than erythrocyte sedimentation rate (ESR) [3].

ISIS 329993 (ISIS-CRP ${ }_{R x}$ ) is an antisense drug targeted to human CRP. ISIS-CRP $P_{R x}$ has been tested in a rodent model of RA (that is, CRP transgenic mice with collageninduced arthritis) and was shown to improve the clinical signs of arthritis [4]. Further, in a previously conducted clinical study in healthy human volunteers $(\mathrm{N}=8)$, whose blood hs-CRP levels ranged from $\geq 2$ to $10 \mathrm{mg} / \mathrm{L}$ on two qualifying examinations within a two-week period, treatment with ISIS-CRP ${ }_{R x}$ achieved significant hs-CRP lowering [4]. The next step in the clinical development process 
was to determine if ISIS-CRP $\mathrm{Rx}_{\mathrm{x}}$ would be effective in reducing hs-CRP in patients with a chronic inflammatory disease, RA.

The objectives of this clinical study were to evaluate the pharmacodynamics, safety and tolerability of ISIS$\mathrm{CRP}_{\mathrm{Rx}}$ in patients with active RA.

\section{Methods}

This Phase II, international, multi-center, double-blind, placebo-controlled, parallel group study in patients with RA was conducted at three sites in Canada and ten sites in Russia with enrollment beginning on 3 October 2011 and ending on 18 December 2012. The Institutional Review Board at each site approved the study protocol and the informed consent (see Additional file 1 for details of the ethical bodies). This study was performed in accordance with globally accepted standards of Good Clinical Practice (as defined in the International Conference on Harmonisation E6 Guidelines for Good Clinical Practice, 1 May 1996), and in agreement with the Declaration of Helsinki and in keeping with local regulations. Prior to screening, all subjects provided written informed consent. The trial was registered with clinicaltrials.gov (www.clinicaltrials.gov) [Identification number: NCT01414101].

\section{Patients}

Patients (18 to 75 years of age) with active RA, as defined by the American Rheumatism Association 1987 revised criteria [5], for at least six months, and a function Class I-III classified according to the American College of Rheumatology 1991 criteria were enrolled [6]. Patients were required to have hs-CRP of $\geq 5 \mathrm{mg} / \mathrm{L}$ at screening (upper limit of normal $>3 \mathrm{mg} / \mathrm{L}$ ) with at least six swollen joints and at least six tender joints, based on a 28-joint count. All patients had received at least three months of methotrexate therapy at a stable dose of $\geq 10 \mathrm{mg}$ unless they could not tolerate that dose. Methotrexate, at stable dose, was continued throughout the study. Other concomitant disease-modifying antirheumatic drugs (DMARDs), non-steroidal anti-inflammatory drugs (NSAIDs) and prednisone at $10 \mathrm{mg}$ daily or less were stable prior to and during the study.

\section{Interventions}

Eligible patients were randomized in an equal ratio to one of the three dose cohorts $\left(100 \mathrm{mg}\right.$ ISIS-CRP $\mathrm{Rx}_{\mathrm{x}}$ or placebo; $200 \mathrm{mg}$ ISIS-CRP $\mathrm{Rx}_{\mathrm{R}}$ or placebo; or $400 \mathrm{mg}$ ISIS$\mathrm{CRP}_{\mathrm{Rx}}$ or placebo). Within each dose cohort, patients were randomized to receive ISIS-CRP $P_{\mathrm{Rx}}$ or placebo in a 3:1 ratio. Enrolled patients received subcutaneous (SC) injections of ISIS-CRP $\mathrm{Rx}_{\mathrm{R}}$ or placebo on Days 1, 3, 5 and 8 and then once weekly for the next 11 weeks.

\section{Outcomes}

\section{Pharmacodynamic}

The effects of treatment with ISIS-CRP $\mathrm{Rx}_{\mathrm{R}}$ versus placebo on hs-CRP and other markers of inflammation (for example, ESR, serum amyloid A (SAA), interleukin-6 (IL-6), tumor necrosis factor alpha (TNF- $\alpha$ ), tumor necrosis factor receptor 1 (TNF-RI), tumor necrosis factor receptor 2 (TNF-RII), fibrinogen and complement) were evaluated throughout the study. During screening, patients had to have at least two hs-CRP measurements taken at least one week apart and no more than one week prior to initiation of dosing and the average of these values was used as the subjects' baseline hs-CRP level. Patient hs-CRP levels and other markers of inflammation were assessed during the treatment and posttreatment evaluation period at regular intervals.

\section{Exploratory activity in RA}

The American College of Rheumatology 20\% improvement criteria (ACR20) is a widely accepted index of improvement in RA [7] that refers to an improvement of at least $20 \%$ in a composite measure of the disease. ACR20 response criteria were assessed at baseline on Day 1 prior to dosing and on Day 36 and Day 92. Disease activity was also assessed using the Disease Activity Score Activity Score Calculator for Rheumatoid Arthritis using the ESR (DAS28-ESR) [8]. DAS28-ESR criteria were assessed at baseline on Day 1 prior to dosing and on Day 36 and Day 92. Patients were considered to be in remission if they had a score of less than 2.6 on the DAS28-ESR.

\section{Safety}

Safety was assessed via adverse events (defined as an adverse event that occurred on or after the first dose of treatment) and clinical laboratory parameters throughout the study.

\section{Statistics \\ Sample size}

It was estimated that the standard deviation of the percent change of hs-CRP was approximately $40 \%$ based on the results of a Phase I study [9]. With 12 subjects in each treatment group, the study was designed to have approximately $74 \%$ power to detect a $45 \%$ difference in hs-CRP between the treated group and the placebo group, assuming the treated group would have a $50 \%$ reduction in hsCRP while the control group would have a $5 \%$ reduction. An alpha level of 0.05 was utilized.

\section{Randomization}

Eligible subjects were randomized equally to one of three dose cohorts (ratio $=1: 1: 1$ ). Each dose cohort was to consist of 16 subjects. Within each dose cohort, subjects were randomized to receive ISIS-CRP $\mathrm{Rx}_{\mathrm{R}}$ or placebo in a 3:1 ratio, 
respectively. Subjects were centrally randomized prior to study drug administration (ISIS-CRP $P_{\mathrm{Rx}}$ or placebo) using an automated system.

\section{Blinding}

The study was blinded with respect to the treatment allocation for subjects, investigators, coordinators and sponsor. The dose cohort was not blinded.

\section{Statistical methods}

The safety population consisted of all patients who were randomized and received at least one dose of study drug. The per protocol population consisted of all subjects who were randomized and received at least $60 \%$ of doses ( $\geq 9$ doses), completed the Day 92 or early termination evaluations and did not have any clinically significant protocol deviations.

Demographic and baseline characteristic information was summarized descriptively for each treatment group. Pharmacodynamic endpoints were summarized using the per protocol population. The effects of treatment (ISIS$\mathrm{CRP}_{\mathrm{Rx}}$ versus placebo) on hs-CRP and other markers of inflammation were evaluated at multiple time points after dosing. Baseline hs-CRP was determined as the average of all of the values during the screening period, prior to dosing on Day 1. In addition, the effects of treatment (ISIS-CRP $_{\mathrm{Rx}}$ versus placebo) were evaluated on the proportion of subjects achieving clinical improvement in RA disease activity at Day 36 and Day 92 compared to baseline using the ACR20 response criteria in the per protocol population.

\section{Results}

\section{Patients}

A total of 51 subjects were randomized in this study (12 placebo, 12 ISIS-CRP $_{R x} 100 \mathrm{mg}, 13$ ISIS-CRP $_{R x} 200 \mathrm{mg}$ and 14 ISIS-CRP $P_{R x} 400 \mathrm{mg}$ ) and 41 subjects completed the treatment (11 placebo, 11 ISIS-CRP $P_{\mathrm{Rx}} 100 \mathrm{mg}$, 9 ISIS$\mathrm{CRP}_{R x} 200 \mathrm{mg}$ and 10 ISIS-CRP $_{R x} 400 \mathrm{mg}$ ) (Additional file 2). Demographics and baseline characteristics were similar across the treatment groups (Table 1).

\section{Medication exposure}

The mean number of doses that patients received was similar across treatment groups in the per protocol popu-

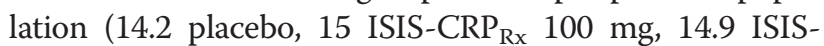
$\mathrm{CRP}_{\mathrm{Rx}} 200 \mathrm{mg}$ and 14.6 ISIS-CRP $\mathrm{Rx} 400 \mathrm{mg}$ ) as was the mean number of days of treatment (81 placebo, 86 ISIS$\mathrm{CRP}_{\mathrm{Rx}} 100 \mathrm{mg}, 86$ ISIS-CRP $_{\mathrm{Rx}} 200 \mathrm{mg}$ and 85 ISIS-CRP $_{\mathrm{Rx}}$ $400 \mathrm{mg})$.

\section{Pharmacodynamics}

Robust, durable, selective and dose dependent reductions of hs-CRP were observed by Day 36, with the effects preserved at Day 92 (Table 2 and Additional file 3). There was a large placebo response (45\% reduction at Day 92$)$ observed in hs-CRP. There were no meaningful differences or trends between placebo and the ISIS-CRP $P_{R x}$ treatment in the other markers of inflammation (that is, fibrinogen, SAA, TNF- $\alpha$, cytokine IL-6, TNF-RI and TNF-RII) at either Day 36 or Day 92. Mean baseline, Day 36 and 92 results are presented in Table 2 for TNF- $\alpha$, IL-6 and SAA.

\section{Exploratory disease activity}

As compared with placebo, there were no statistically significant effects seen with ISIS-CRP $P_{\mathrm{Rx}}$ on disease symptoms as assessed via ACR20, ACR50 or DAS28-ESR (Table 2) at Day 36 or Day 92.

\section{Safety}

ISIS-CRP ${ }_{\mathrm{Rx}}$ was generally safe and well tolerated with no serious infections due to treatment. There were similar numbers of patients who reported treatment emergent adverse events (TEAEs) across the treatment groups (8/ 12 placebo, 7/12 ISIS-CRP $P_{R x} 100 \mathrm{mg}, 11 / 13$ ISIS-CRP $P_{R x}$ $200 \mathrm{mg}$ and 12/14 ISIS-CRP $\mathrm{Rx} 400 \mathrm{mg}$ ) (Table 3). There were a similar number of infections reported in each group (Table 3). Infections were reported as mostly mild in severity with only a few reported as moderate (Table 3 ).

There were seven patients who discontinued treatment either due to TEAEs or meeting the requirements for one of the stopping rules. One patient on ISIS-CRP $P_{R x}$ $200 \mathrm{mg}$ discontinued treatment due to fatal pulmonary edema which also met the criteria for a serious adverse event (SAE). This patient had known atherosclerotic vascular disease. The investigator rated this as possibly related to study drug. Two patients discontinued treatment due to worsening of RA symptoms. One was on ISIS-

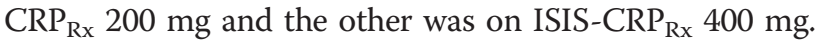
Three patients (one placebo, one ISIS-CRP $\mathrm{Rx}_{\mathrm{x}} 200 \mathrm{mg}$ and

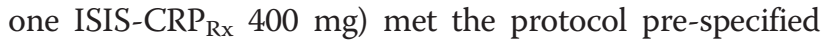
stopping criteria for renal function (that is, serum creatinine $(\mathrm{Cr})$ increase $>0.2 \mathrm{mg} / \mathrm{dL})$. However, the absolute serum $\mathrm{Cr}$ levels remained within the normal limits for all of these patients. One patient on ISIS-CRP ${ }_{R x} 200 \mathrm{mg}$ discontinued treatment due to an exacerbation of chronic calculous cholecystitis although this was considered unlikely related to study drug by the investigator.

\section{Discussion}

ISIS 329993 (ISIS-CRP ${ }_{\mathrm{Rx}}$ ) is a novel approach to reducing the inflammatory marker CRP and to potentially down regulate inflammation. As an antisense drug targeting human CRP it was hypothesized that ISIS-CRP $\mathrm{Rx}_{\mathrm{R}}$ should be non-immunosuppressant and it, therefore, might be a valuable adjunct to standard DMARDs, or even biologic DMARDs. Previous studies of ISIS-CRP $P_{R x}$ 
Table 1 Baseline demographics and disease characteristics

\begin{tabular}{|c|c|c|c|c|}
\hline Demographics (Safety Population) & Number $=12$ & $\begin{array}{l}\text { ISIS-CRP }_{\mathrm{Rx}} \\
100 \mathrm{mg} \\
\text { Number }=12\end{array}$ & $\begin{array}{l}\text { ISIS-CRP }_{\mathrm{Rx}} \\
200 \mathrm{mg} \\
\text { Number = 13 }\end{array}$ & $\begin{array}{l}\text { ISIS-CRP }_{\mathrm{Rx}} \\
400 \mathrm{mg} \\
\text { Number }=14\end{array}$ \\
\hline \multicolumn{5}{|l|}{ Race } \\
\hline White, number (\%) & $12(100)$ & $12(100)$ & $13(100)$ & $14(100)$ \\
\hline \multicolumn{5}{|l|}{ Sex } \\
\hline Female, number (\%) & $12(100)$ & $9(75)$ & $8(61.5)$ & $13(92.9)$ \\
\hline Age, years, mean (SD) & $64.1(4.25)$ & $56.08(13.14)$ & $58.69(9.81)$ & $62.93(7.97)$ \\
\hline Weight, kg, mean (SD) & 72.29 (13.39) & $74.05(19.81)$ & $73.75(16.43)$ & 72.19 (13.39) \\
\hline $\mathrm{BMl}, \mathrm{kg} / \mathrm{m}^{2}$, mean $(\mathrm{SD})$ & $28.36(4.97)$ & $27.27(5.04)$ & $25.78(4.93)$ & $26.84(3.63)$ \\
\hline Duration of RA since 1st diagnosis, years, mean (SD) & $18.6(15.8)$ & $6.3(8.1)$ & $8.8(7.8)$ & $18.3(12.4)$ \\
\hline \multicolumn{5}{|l|}{ ACR Functional Class, number (\%) } \\
\hline Class I & $1(8.3)$ & $1(8.3)$ & 0 & $1(7.1)$ \\
\hline Class II & $6(50.0)$ & $8(66.7)$ & $7(53.8)$ & $7(50.0)$ \\
\hline Class III & $5(41.7)$ & $3(25.0)$ & $6(46.2)$ & $6(42.9)$ \\
\hline Morning stiffness, minutes, mean (SD) & $61.7(29.3)$ & $98.3(97.5)$ & $90.4(59.0)$ & $106.4(75.9)$ \\
\hline Disease Characteristics (Per Protocol Population) & Number $=11$ & Number $=10$ & Number $=8$ & Number $=11$ \\
\hline Westergren ESR, mm/hour, mean (SD) & $41.0(27.7)$ & $47.6(27.3)$ & $37.4(14.0)$ & $44.5(31.1)$ \\
\hline hsCRP, mg/L, mean (SD) & $22.4(18.1)$ & $29.7(29.0)$ & $35.2(36.4)$ & $19.3(13.6)$ \\
\hline Swollen joint count, mean (SD) & $12.5(5.6)$ & $9.7(6.4)$ & $9.8(4.5)$ & $8.3(2.2)$ \\
\hline Tender joint count, mean (SD) & $17.6(8.3)$ & $15.9(8.0)$ & $14.8(7.5)$ & $14.1(5.7)$ \\
\hline DAS28-ESR, mean (SD) & $6.66(0.6)$ & $6.36(1.1)$ & $6.27(0.7)$ & $6.23(0.9)$ \\
\hline $\mathrm{HAQ}$, mean (SD) & $1.67(0.6)$ & $1.50(0.7)$ & $1.58(0.6)$ & $1.73(0.6)$ \\
\hline Patient's pain assessment, mean (SD) & $66.82(16.4)$ & $54.50(16.6)$ & $59.75(13.2)$ & $61.55(18.4)$ \\
\hline Patient's global assessment of disease activity, mean (SD) & $2.64(0.7)$ & $2.30(0.7)$ & $2.38(0.5)$ & $2.45(0.5)$ \\
\hline Physician's global assessment of disease activity, mean (SD) & $2.18(0.4)$ & $2.10(0.7)$ & $2.50(0.5)$ & $2.36(0.5)$ \\
\hline
\end{tabular}

ACR, American College of Rheumatology; BMI, body mass index; DAS28-ESR, disease activity score 28-erythrocyte sedimentation rate; ESR, erythrocyte sedimentation rate; $\mathrm{HAQ}$, Health Assessment Questionnaire; hsCRP, high sensitivity C-reactive protein; percentage calculated as $100 \times \mathrm{n} / \mathrm{N}$; $\mathrm{SD}$, standard deviation.

in rodent models of RA (that is, CRP transgenic mice with collagen-induced arthritis) demonstrated improvement in the clinical signs of arthritis [4]. Subsequently, initial clinical studies in healthy human volunteers demonstrated that treatment with ISIS-CRP $\mathrm{Rx}_{\mathrm{x}}$ achieved significant hs-CRP lowering [4]. Before embarking on a large RA study to definitively address the question of clinical improvement in symptoms and signs, it was decided to first explore whether this approach would reproducibly decrease hs-CRP in RA patients and to assess its potential impact on other markers of inflammation. Given the results of this study, ISIS-CRP $\mathrm{Rx}_{\mathrm{x}}$ has demonstrated that the antisense oligonucleotide platform can selectively reduce circulating levels of hs-CRP. The results at 36 days demonstrate separation between dosing cohorts with respect to percent change in hsCRP and achieved statistical significance at the highest dose, with a $76.7 \%$ reduction in hs-CRP in the ISIS $\mathrm{CRP}_{\mathrm{Rx}} 400 \mathrm{mg}$ arm compared with a $14.4 \%$ reduction in the placebo arm at Day $36(P=0.0015)$. However, by
92 days this response does not appear as statistically robust given the significant decreases in hs-CRP seen in the placebo group. It is unclear what drove the decreases in hs-CRP in the placebo group but it could be speculated that it was in part regression to the mean, and possibly related to increased compliance with background regimens that can also be seen in clinical trials as a consequence of closer follow-up.

It remains unclear whether ISIS-CRP $P_{R x}$ is truly antiinflammatory in an active human disease state. Despite the encouraging data in the animal models, there was little change in the other inflammatory markers studied in this trial including ESR, SAA, IL-6, TNF- $\alpha$, fibrinogen and complement. This invites the question as to whether inflammation was down regulated or whether the drug was simply effective at blocking a surrogate marker of inflammation. Additionally, the exploratory endpoints of ACR20/50 and DAS28-ESR were frankly difficult to interpret in the setting of such small numbers and such high placebo response rates. That said, the reductions 
Table 2 Inflammation markers and disease activity outcomes (per protocol population)

\begin{tabular}{|c|c|c|c|c|c|c|c|}
\hline & $\begin{array}{l}\text { Placebo } \\
\text { Nimber }=11\end{array}$ & $\begin{array}{l}\text { ISIS-CRP } \\
100 \mathrm{mg} \\
\text { Number }=10\end{array}$ & & $\begin{array}{l}\text { ISIS-CRP }_{\mathrm{Rx}} \\
200 \mathrm{mg} \\
\text { Number = 8 }\end{array}$ & p-value & $\begin{array}{l}\text { ISIS-CRP } \\
400 \mathrm{mg} \\
\text { Number }=11\end{array}$ & \\
\hline INFLAMMATION MARKERS & & & $P$-value ${ }^{a}$ & & $P$-value ${ }^{a}$ & & $P$-value ${ }^{a}$ \\
\hline hs-CRP (mg/L) & Number $=11$ & Number $=10$ & & Number $=8$ & & Number $=11$ & \\
\hline \multirow[t]{2}{*}{ Baseline, mean (SD) } & $22.4(18.1)$ & $29.7(29.0)$ & 0.97 & $35.2(36.4)$ & 0.24 & $19.3(13.6)$ & 0.65 \\
\hline & Number $=10$ & Number $=10$ & & Number $=8$ & & Number $=11$ & \\
\hline Day 36, mean (SD) & $16.4(12.3)$ & $16.9(16.2)$ & 0.85 & $19.1(25.9)$ & 0.57 & $4.26(4.2)$ & 0.004 \\
\hline Day 36, mean change from baseline (SD) & $-6.8(15.6)$ & $-12.8(20.9)$ & 0.85 & $-16.1(12.1)$ & 0.15 & $-15.0(11.5)$ & 0.07 \\
\hline \multirow{2}{*}{$\begin{array}{l}\text { Day } 36, \text { percentage change from } \\
\text { baseline, mean (SD) }\end{array}$} & $-14.4(66.3)$ & $-19.5(63.6)$ & 0.97 & $-56.6(26.2)$ & 0.10 & $-76.7(16.4)$ & 0.002 \\
\hline & Number $=11$ & Number $=10$ & & Number $=8$ & & Number $=11$ & \\
\hline Day 92, mean (SD) & $10.8(10.5)$ & $15.0(19.1)$ & 0.93 & $12.0(10.7)$ & 0.89 & $5.6(5.2)$ & 0.22 \\
\hline Day 92, mean change from baseline (SD) & $-11.6(17.3)$ & $-14.7(18.9)$ & 0.81 & $-23.2(28.2)$ & 0.13 & $-13.7(13.3)$ & 0.80 \\
\hline \multirow{2}{*}{$\begin{array}{l}\text { Day 92, percentage change from baseline, } \\
\text { mean (SD) }\end{array}$} & $-44.5(60.2)$ & $-33.8(82.3)$ & 0.86 & $-66.9(23.0)$ & 0.49 & $-64.4(38.6)$ & 0.19 \\
\hline & & & $P$-value ${ }^{a}$ & & $P$-value ${ }^{a}$ & & $P$-value ${ }^{a}$ \\
\hline TNF-a (pg/mL) & Number $=11$ & Number $=10$ & & Number $=8$ & & Number $=11$ & \\
\hline \multirow[t]{2}{*}{ Baseline, mean (SD) } & $15.6(0.0)$ & $15.6(0.0)$ & 1.00 & $15.6(0.0)$ & 1.00 & $15.6(0.0)$ & 1.00 \\
\hline & Number $=10$ & Number $=10$ & & Number $=8$ & & Number $=11$ & \\
\hline \multirow[t]{2}{*}{ Day 36, mean (SD) } & $15.6(0.0)$ & $15.6(0.0)$ & 1.00 & $15.6(0.0)$ & 1.00 & $15.6(0.0)$ & 1.00 \\
\hline & Number $=11$ & Number $=10$ & & Number $=8$ & & Number $=11$ & \\
\hline \multirow[t]{2}{*}{ Day 92, mean (SD) } & $15.6(0.0)$ & $15.6(0.0)$ & 1.00 & $15.9(0.8)$ & 0.42 & $16.0(1.4)$ & 1.00 \\
\hline & & & $P$-value ${ }^{a}$ & & $P$-value ${ }^{a}$ & & $P$-value ${ }^{a}$ \\
\hline IL-6 (pg/mL) & Number $=10$ & Number $=9$ & & Number $=8$ & & Number $=11$ & \\
\hline \multirow[t]{2}{*}{ Baseline, mean (SD) } & $24.6(20.3)$ & $46.2(45.3)$ & 0.24 & $35.7(32.9)$ & 0.57 & $25.6(26.1)$ & 0.92 \\
\hline & Number $=10$ & Number $=10$ & & Number $=7$ & & Number $=11$ & \\
\hline \multirow[t]{2}{*}{ Day 36, mean (SD) } & $21.4(19.8)$ & $35.7(32.5)$ & 0.68 & $27.9(21.6)$ & 0.67 & $28.3(42.1)$ & 0.97 \\
\hline & Number $=11$ & Number $=10$ & & Number $=7$ & & Number $=11$ & \\
\hline \multirow[t]{2}{*}{ Day 92, mean (SD) } & $30.3(41.6)$ & $32.1(28.1)$ & 0.65 & $83.1(142.6)$ & 0.20 & $53.5(62.3)$ & 0.30 \\
\hline & & & $P$-value ${ }^{a}$ & & $P$-value ${ }^{a}$ & & $P$-value ${ }^{a}$ \\
\hline Serum Amyloid A (mg/L) & Number $=11$ & Number $=10$ & & Number $=8$ & & Number $=11$ & \\
\hline \multirow[t]{2}{*}{ Baseline, mean (SD) } & $75.5(167.4)$ & $67.9(85.4)$ & 0.92 & $171.1(255.1)$ & 0.35 & $35.5(44.4)$ & 0.62 \\
\hline & Number $=10$ & Number $=10$ & & Number $=8$ & & Number = 11 & \\
\hline \multirow[t]{2}{*}{ Day 36, mean (SD) } & $33.9(41.2)$ & $45.8(38.4)$ & 0.44 & $105.1(192.2)$ & 0.24 & $48.3(86.4)$ & 0.67 \\
\hline & Number $=11$ & Number $=10$ & & Number $=8$ & & Number $=11$ & \\
\hline Day 92, mean (SD) & $24.8(30.0)$ & $42.6(39.2)$ & 0.28 & $96.8(112.0)$ & 0.03 & $88.9(98.9)$ & 0.07 \\
\hline \multicolumn{8}{|l|}{ DISEASE ACTIVITY OUTCOMES } \\
\hline \multicolumn{3}{|l|}{$A C R$} & $P$-value ${ }^{\mathrm{b}}$ & & $P$-value ${ }^{b}$ & & $P$-value ${ }^{b}$ \\
\hline Day 36, number (\% of responders) & Number $=10$ & Number $=10$ & & Number $=8$ & & Number $=11$ & \\
\hline ACR20\% by hs-CRP & $2(20.0)$ & $3(30.0)$ & 1.000 & 0 & 0.477 & $4(36.4)$ & 0.635 \\
\hline ACR20\% by ESR & $2(20.0)$ & $3(30.0)$ & 1.000 & 0 & 0.477 & $3(27.3)$ & 1.000 \\
\hline ACR50 by hs-CRP & $1(10.0)$ & $2(20.0)$ & 1.000 & 0 & 1.000 & $1(9.1)$ & 1.000 \\
\hline ACR50 by ESR & 0 & $2(20.0)$ & 0.474 & 0 & & 0 & \\
\hline
\end{tabular}


Table 2 Inflammation markers and disease activity outcomes (per protocol population) (Continued)

\begin{tabular}{|c|c|c|c|c|c|c|c|}
\hline & & & $P$-value ${ }^{\mathrm{b}}$ & & $P$-value ${ }^{\mathbf{b}}$ & & $P$-value ${ }^{\mathbf{b}}$ \\
\hline Day 92, number (\% of responders) & Number $=11$ & Number $=10$ & & Number $=8$ & & Number $=11$ & \\
\hline ACR20 by hs-CRP & $6(54.5)$ & $5(50.0)$ & 1.000 & $1(12.5)$ & 0.147 & $4(36.4)$ & 0.392 \\
\hline ACR20 by ESR & $6(54.5)$ & $4(40.0)$ & 0.670 & $1(12.5)$ & 0.147 & $4(36.4)$ & 0.392 \\
\hline ACR50 by hs-CRP & $2(18.2)$ & $2(20.0)$ & 1.000 & 0 & 0.485 & $3(27.3)$ & 1.000 \\
\hline ACR50 by ESR & $1(9.1)$ & $2(20.0)$ & 0.586 & 0 & 1.000 & $2(18.2)$ & 1.000 \\
\hline \multirow[t]{2}{*}{ DAS28-ESR } & & & $P$-value ${ }^{\mathrm{a}}$ & & $P$-value ${ }^{a}$ & & $P$-value ${ }^{a}$ \\
\hline & Number $=11$ & Number $=10$ & & Number $=8$ & & Number $=11$ & \\
\hline \multirow[t]{2}{*}{ Baseline, mean (SD) } & $6.7(0.6)$ & $6.4(1.1)$ & 0.4298 & $6.3(0.7)$ & 0.3352 & $6.2(0.9)$ & 0.2489 \\
\hline & Number $=10$ & Number $=10$ & & Number $=8$ & & Number $=11$ & \\
\hline Day 36, mean (SD) & $6.2(1.1)$ & $5.4(1.5)$ & 0.1097 & $5.8(1.1)$ & 0.4974 & $5.2(1.2)$ & 0.0572 \\
\hline Day 36, mean change from baseline (SD) & $-0.5(0.6)$ & $-1.0(0.9)$ & 0.2176 & $-0.4(0.7)$ & 0.8286 & $-1.0(0.8)$ & 0.0986 \\
\hline \multirow{2}{*}{$\begin{array}{l}\text { Day 36, percentage change from baseline, } \\
\text { mean(SD) }\end{array}$} & $-8.0(10.7)$ & $-16.3(16.1)$ & 0.1431 & $-7.1(11.2)$ & 0.8968 & $-16.8(12.7)$ & 0.1321 \\
\hline & Number $=11$ & Number $=10$ & & Number $=8$ & & Number $=11$ & \\
\hline Day 92, mean (SD) & $5.3(1.6)$ & $5.2(1.5)$ & 0.8711 & $5.6(1.1)$ & 0.7164 & $5.1(1.2)$ & 0.6880 \\
\hline Day 92, mean change from baseline (SD) & $-1.3(1.2)$ & $-1.1(1.1)$ & 0.6833 & $-0.7(0.7)$ & 0.2422 & $-1.1(1.3)$ & 0.6887 \\
\hline $\begin{array}{l}\text { Day } 92 \text {, percentage change from baseline, } \\
\text { mean (SD) }\end{array}$ & $-20.7(19.7)$ & $-17.5(18.1)$ & 0.6873 & $-11.7(11.1)$ & 0.2982 & $-16.9(21.2)$ & 0.6237 \\
\hline
\end{tabular}

Table 3 Adverse events (safety population)

\begin{tabular}{|c|c|c|c|c|}
\hline & $\begin{array}{l}\text { Placebo } \\
\text { Number }=12\end{array}$ & $\begin{array}{l}\text { ISIS-CRP }_{\mathrm{Rx}} \\
100 \mathrm{mg} \\
\text { Number = } 12\end{array}$ & $\begin{array}{l}\text { ISIS-CRP } \\
200 \mathrm{mg} \\
\text { Number = } 13\end{array}$ & $\begin{array}{l}\text { ISIS-CRP }_{\mathrm{Rx}} \\
400 \mathrm{mg} \\
\text { Number }=14\end{array}$ \\
\hline Overall adverse events, $\mathrm{n}(\%)$ & $8(66.7)$ & $7(58.3)$ & $11(84.6)$ & $12(85.7)$ \\
\hline \multicolumn{5}{|c|}{$\begin{array}{l}\text { Adverse events with incidence } 10 \% \text { or greater in all ISIS-CRPRx } \\
\text { dosed patients by treatment excluding adverse events at } \\
\text { the injection site, }\end{array}$} \\
\hline Rheumatoid arthritis, number (\%) & $1(8.3)$ & $1(8.3)$ & $2(15.4)$ & $2(14.3)$ \\
\hline Blood creatinine increased, number (\%) & $1(8.3)$ & $2(16.7)$ & $1(7.7)$ & $1(7.1)$ \\
\hline Adverse events-infections, number (\%) & $4(33.3)$ & $1(8.3)$ & $2(15.4)$ & $4(28.6)$ \\
\hline Nasopharyngitis, number (severity) & 1 (moderate) & 1 (mild) & 1 (mild) & 1 (moderate) \\
\hline Upper respiratory infection, number (severity) & 0 & 0 & 0 & 1 (mild) \\
\hline Rhinitis, number (severity) & 1 (mild) & 0 & 0 & 0 \\
\hline Bronchitis, number (severity) & 2 (moderate) & 0 & 1 (moderate) & 0 \\
\hline Urinary tract infection/cystitis, number (severity) & 0 & 1 (mild) & 1 (moderate) & 2 (mild) \\
\hline Foot infection, number (severity) & 0 & 0 & 0 & 1 (mild) \\
\hline Pyelonephritis chronic, number (severity) & 1 (mild) & 0 & 0 & 0 \\
\hline \multicolumn{5}{|l|}{ Adverse events-injection site reactions } \\
\hline Overall, number (\%) & 0 & $3(25)$ & $3(23)$ & $3(21)$ \\
\hline Erythema, number (\%) & 0 & $3(25)$ & $3(23)$ & $3(21)$ \\
\hline Pruritus, number (\%) & 0 & $1(8)$ & 0 & 0 \\
\hline Pain, number (\%) & 0 & 0 & 0 & $1(7)$ \\
\hline Swelling, number (\%) & 0 & 0 & 0 & $1(7)$ \\
\hline
\end{tabular}


observed in the clinical measures of disease activity were not of sufficient magnitude to offer significant benefit over the currently available drugs for treatment of RA.

It appeared that ISIS-CRP $P_{R x}$ was well tolerated in a population with active autoimmune/inflammatory disease. However, the small sample size limits the ability to detect uncommon safety signals.

There were some limitations to this study, most notably the overall size and powering together with the very large placebo response rate limited the conclusions that could be drawn. However, even with a small sample size, the lack of any discernible effect on other markers of inflammation coupled with the very modest clinical changes make it unlikely that ISIS-CRP $\mathrm{Rx}_{\mathrm{x}}$ will be an adequately effective drug for treatment of rheumatoid arthritis when compared with other currently available and experimental therapies.

\section{Conclusions}

In this study, ISIS-CRP $\mathrm{Rx}_{\mathrm{x}}$ selectively reduced hs-CRP in a dose-dependent manner, and was well-tolerated in patients with RA. Its utility as a therapy in RA remains unclear. There may be other chronic inflammatory conditions in which CRP has a causative or contributory role; so additional studies in other diseases may be warranted given the tolerability of ISIS-CRP $\mathrm{Rx}_{\mathrm{x}}$, the lack of obvious immune suppression and the potential for combination with other agents.

\section{Additional files}

Additional file 1: Listing of Ethics Committees and Institutional Review Boards (IRB).

Additional file 2: Flow of patients through study. CONSORT patient disposition diagram.

Additional file 3: Change in hs-CRP by treatment group. Panel $A$ shows mean absolute change from baseline $(\mathrm{mg} / \mathrm{L})$ in hs-CRP at Day 36 and Day 92 by dose group. Panel B shows mean percent change from baseline in hs-CRP at Day 36 and Day 92 by dose group).

\section{Abbreviations}

ACR20: American College of Rheumatology 20\% improvement criteria; ACR50: American College of Rheumatology 50\% improvement criteria; Cr: creatinine; CRP: C-reactive protein; DAS28-ESR: Disease Activity Score Activity Score Calculator for Rheumatoid Arthritis using the ESR; DMARD: disease-modifying antirheumatic drugs; ESR: erythrocyte sedimentation rate; Hs-CRP: high sensitivity C-reactive protein assay; NSAIDs: non-steroidal anti-inflammatory drugs; RA: rheumatoid arthritis; SAA: serum amyloid A; TEAEs: treatmemt emergent adverse effects; TNF-R2: tumor necrosis factor receptor 2; TNF-RI: tumor necrosis factor receptor 1 ; TNF-a: tumor necrosis factor alpha.

\section{Competing interests}

Marshelle S. Warren, Steven G. Hughes, Walter Singleton, and Mason Yamashita are employees of ISIS Pharmaceuticals, Inc. Marshelle S. Warren, Steven G. Hughes, Walter Singleton, and Mason Yamashita have no non-financial competing interests. Mark C. Genovese declares that he has no competing interests.

\section{Authors' contributions}

MSW was involved in the execution of the study, interpretation of the data, drafting the manuscript as well as review and critique of the manuscript. SH was involved in the conception, organization and execution of the study, interpretation of the data, drafting of the manuscript, review and critique of the statistical analysis and review and critique of the manuscript. WS was involved in the conception and organization of this study and drafting, review and critique of the manuscript. MY was involved in the execution of the study, he reviewed and critiqued the statistical analysis plan and was involved in the interpretation of the data and drafting, review and critique of the manuscript. MCG was involved in the review and critique of the statistical analysis, interpretation of the data and drafting, review and critique of the manuscript. All authors take public responsibility for the content of the manuscript. Each author agrees to be accountable for all aspects of the work in ensuring that questions related to the accuracy or integrity of any part of work are appropriately investigated and resolved. All authors read and approved the final manuscript.

\section{Acknowledgements}

The authors thank Starr Grundy of SD Scientific for her assistance in preparation of the manuscript. The study was funded by Isis Pharmaceuticals, Inc.

\section{Author details}

'Isis Pharmaceuticals, Inc, 2855 Gazelle Court, Carlsbad, CA 92010, USA.

${ }^{2}$ Stanford University, 1000 Welch Rd Palo, Alto, CA 94304, USA.

Received: 17 July 2014 Accepted: 20 February 2015

Published online: 19 March 2015

\section{References}

1. Emery P, Gabay C, Kraan M, Gomez-Reino J. Evidence-based review of biologic markers as indicators of disease progression and remission in rheumatoid arthritis. Rheumatol Int. 2007;27:793-806.

2. Molenaar ET, Voskuyl AE, Familian A, van Mierlo GJ, Dijkmans BA, Hack CE. Complement activation in patients with rheumatoid arthritis mediated in part by C-reactive protein. Arthritis Rheum. 2001;44:997-1002.

3. Dessein $\mathrm{PH}$, Joffe $\mathrm{BI}$, Stanwix AE. High sensitivity C-reactive protein as a disease activity marker in rheumatoid arthritis. J Rheumatol. 2004;31:1095-7.

4. Jones NR, Pegues MA, McCrory MA, Singleton W, Bethune C, Baker BF, et al. A selective inhibitor of human C-reactive protein translation is efficacious in vitro and in C-reactive protein transgenic mice and humans. Mol Ther Nucleic Acids. 2012;1:e52

5. Arnett FC, Edworthy SM, Bloch DA, McShane DJ, Fries JF, Cooper NS, et al. The American Rheumatism Association 1987 revised criteria for the classification of rheumatoid arthritis. Arthritis Rheum. 1988;31:315-24.

6. Hochberg MC, Chang RW, Dwosh I, Lindsey S, Pincus T, Wolfe F. The American College of Rheumatology 1991 revised criteria for the classification of global functional status in rheumatoid arthritis. Arthritis Rheum. 1992;35:498-502.

7. Felson DT, Anderson JJ, Boers M, Bombardier C, Furst D, Goldsmith C, et al. American College of Rheumatology. Preliminary definition of improvement in rheumatoid arthritis. Arthritis Rheum. 1995;38:727-35.

8. Prevoo ML, Van 't Hof MA, Kuper HH, van Leeuwen MA, van de Putte LB, van Riel PL. Modified disease activity scores that include twenty-eight-joint counts. Development and validation in a prospective longitudinal study of patients with rheumatoid arthritis. Arthritis Rheum. 1995;38:44-8.

9. ISIS Pharmaceuticals Inc. Data on File. \Visis.local \ogroups\cdmstats\329993 ICS1 \testdir\CohortIIIprograms\thsCRPetCIII.sas DCHEN SASv9.2 (23MAR2011 14:13) Data Download: 21 March 2011. 